\title{
Evolving Treatment Strategies for Triple-Negative Breast Cancer
}

Presented by Melinda Telli, MD

\begin{abstract}
Although not yet practice changing in early-stage triple-negative breast cancer, growing evidence suggests that neoadjuvant platinum-based therapy is active and that some patients may benefit from such an approach. Recent randomized phase III data suggests that carboplatin has comparable efficacy to docetaxel as first-line therapy in unselected advanced triple-negative breast cancer. In both settings, the efficacy of such treatment appears to be influenced by $B R C A 1 / 2$ mutation status, with carriers of these mutations experiencing higher response rates. To optimize patient selection, biomarkers of response need to be incorporated into randomized clinical trials and validated. In addition to $B R C A 1 / 2$, it is likely that measures of genomic instability and other germline biomarkers beyond $B R C A 1 / 2$ may be associated with therapeutic sensitivity. (J Natl Compr Canc Netw 2015;13:652-654)
\end{abstract}

"The standard treatment of triple-negative breast cancer has not changed significantly in over 10 years," admitted Melinda Telli, MD, Assistant Professor of Medicine in the Division of Oncology at Stanford University School of Medicine, and Medical Oncologist at the Stanford Cancer Institute. She currently serves as a member of the NCCN Guidelines Panel for Breast Cancer. Additionally, the selective use and targeting of our available cytotoxics have not been optimized, "but we are starting to see some changes here," she added.

During her presentation at the NCCN 20th Annual Conference, Dr. Telli briefly explored the studies behind recent renewed interest in the use of platinum

Presented by Melinda Telli, MD, Assistant Professor of Medicine, Division of Oncology, Stanford University School of Medicine, and Medical Oncologist, Stanford Cancer Institute, Palo Alto, California. Dr. Telli has disclosed that she has served as a consultant for Vertex Pharmaceuticals Inc.

Correspondence: Melinda Telli, MD, Stanford Cancer Institute, Stanford University School of Medicine, 875 Blake Wilbur Drive, MC 5826, Room 2241, Stanford, CA 94305-5826.

E-mail: mtelli@stanford.edu chemotherapy for breast cancer, suggesting that women with triple-negative and BRCA1/2-mutant breast cancer may be more likely to benefit from a DNA repair defect-targeted approach. In addition, she looked at the difference in response to carboplatin versus docetaxel in the metastatic setting based on BRCA1/2-mutation status and shared the promising early results with the immunotherapeutic agent pembrolizumb in some women with advanced disease.

\section{Neoadjuvant Use of Platinum}

Standard neoadjuvant therapy for a premenopausal woman with early-stage invasive ductal carcinoma who has triple-negative disease and no BRCA1 or BRCA2 mutation identified would be an anthracycline and taxanebased regimen, such as the regimen of dose-dense doxorubicin and cyclophosphamide followed by dose-dense paclitaxel, according to Dr. Telli. However, she added, for a woman with the same type of cancer who has triple-negative disease and a germline BRCA1 mutation, the question of whether to include a platinum in the neoadjuvant setting is under consideration.

Several years ago, the neoadjuvant use of singleagent cisplatin in BRCA1 mutation carriers with primarily triple-negative breast cancer generated much enthusiasm and raised many questions, Dr. Telli revealed. Retrospective data have been mixed as to the difference in response to standard anthracycline/taxane-based therapy based on the presence or absence of a germline BRCA1/2 mutation. While the initial interest in platinum was greatest for BRCA1/2 mutation carriers, the recently reported randomized phase II GeparSixto and CALGB 40603 trials took a different approach and set out to evaluate the role of carboplatin as an "add-on" to standard neoadjuvant chemotherapy in women with unselected triple-negative breast cancer. ${ }^{1,2}$ 
According to the German GeparSixto study, ${ }^{1}$ the inclusion of carboplatin improved the pathologic complete response ( $\mathrm{pCR}$ ) rate compared with paclitaxel, nonpegylated liposomal doxorubicin, and bevacizumab used alone among those patients with triple-negative breast cancer. Although a modest, but not statistically significant, improvement was seen in pCR rates with the use of carboplatin in the overall population, a $16 \%$ ( $53 \%$ vs $37 \%$ ) improvement was seen in the triple-negative breast cancer subgroup, which was highly statistically significant, added Dr. Telli. In the United States, results of the CALGB 40603 trial $^{2}$ showed a 13\% statistically significant improvement in the $\mathrm{pCR}$ rate in the breast and axilla with the addition of carboplatin to standard paclitaxel, doxorubicin, and cyclophosphamide with or without bevacizumab. Whether the addition of carboplatin to standard neoadjuvant chemotherapy will ultimately improve relapse-free or overall survival is still unknown.

In a follow-up to the GeparSixto trial, ${ }^{3}$ the investigators found that patients treated with carboplatin who carried BRCA1/2 and RAD50/51C germline mutations seemed to experience a higher $\mathrm{pCR}$ rate than those without these mutations. Furthermore, added Dr. Telli, those patients who lacked a germline mutation in BRCA1/2 and RAD50/51C, but had a positive family history of breast or ovarian cancer, seemed to also benefit substantially from the addition of carboplatin. So while germline BRCA1/2 and RAD50/51C mutation status emerged as a potential biomarker of platinum response in this study, other patients lacking these mutations but with a family history of breast or ovarian cancer also benefited, she noted. In addition to BRCA1 and BRCA2, many other genes in the homologous recombination pathway have been implicated in familial breast cancer, and these germline alterations may be relevant in the prediction of platinum sensitivity.

Finally, the recently completed single-arm phase II PrECOG 0105 study evaluated gemcitabine and carboplatin plus iniparib in the neoadjuvant setting for triple-negative and BRCA1/2 mutationassociated breast cancer. ${ }^{4}$ Dr. Telli reported that in the intent-to-treat population, the $\mathrm{pCR}$ rates were higher in those with BRCA1/2 mutations-and higher still in those with both triple-negative and BRCA1/2-mutant disease-than in those who were not carriers of these mutations $(47 \%, 56 \%$, and 33\%, respectively). In addition, "The combination of the homologous recombination deficiency (HRD) assay and BRCA1/2 status might provide a good ability to discriminate responders from nonresponders," Dr. Telli noted. The HRD assay provides a measure of global genomic instability and reflects the accumulation of genetic changes in tumors with underlying DNA repair deficiency. In PrECOG 0105, patients with and without a BRCA1/2 mutation, but with a high HRD score, benefited greatest from this platinum-based approach.

\section{Role of Platinum for Metastatic Disease}

For many years, randomized data comparing platinum with other standard chemotherapies for metastatic triple-negative disease have been limited. Therefore, Dr. Telli considered the recently reported phase III TNT trial "extremely important" and briefly discussed some of its key findings. ${ }^{5}$

In this United Kingdom study involving women with metastatic or recurrent locally advanced triplenegative or BRCA1/2-positive breast cancer, 31\% responded to treatment with carboplatin compared with 36\% who responded to docetaxel. These results were not significantly different $(P=.44)$. However, a significant advantage in objective response rate (68\% vs $33 \% ; P=.03)$ and progression-free survival was seen with carboplatin over docetaxel in those who had a germline BRCA1/2 mutation. Moreover, as for toxicity, "Patients treated with docetaxel had significantly higher rates of febrile neutropenia and neuropathy, whereas in patients treated with carboplatin, vomiting appeared to be somewhat more common," explained Dr. Telli.

Furthermore, she said, "disappointingly, there did not appear to be any advantage in this trial with carboplatin among patients with a high HRD score. Certainly, this has raised some concerns about whether it is valid to assess HRD on a primary tumor and use that information to potentially predict response in the metastatic setting," Dr. Telli added.

\section{Glimpse at Immunotherapy}

Tumors are immunologically unique because of tumor-specific mutations, although they represent only a fraction of the total number of mutations in the tumor, declared Dr. Telli. In addition, she added, these 
Telli

tumor-specific mutations are thought to be able to produce "neoantigens," which can be recognized by the immune system. Moreover, she raised the question of whether BRCA1/2-mutant tumors are more immunogenic because of higher levels of mutations. The issue of whether this increased burden of mutations and resulting neoantigens renders these tumors more amenable to immunotherapy represents a "hot topic of research," she stated.

Dr. Telli concluded with some early clinical data on 2 immune therapies in the setting of advanced triple-negative breast cancer: pembrolizumab, which has recently been approved in melanoma, and MPDL3280A. In the phase IB KEYNOTE-012 trial, ${ }^{6}$ nearly $60 \%$ of screened patients tested positive for programmed death-ligand 1 (PD-L1). Early results have shown an objective response rate of $18.5 \%$ and a median progression-free survival of 1.9 months in a heavily pretreated population.

In the study of MPDL3280A, ${ }^{7} 23 \%$ of patients screened tested positive for PD-L1. Thus far, the objective response rate with this investigational agent is $33 \%$. The study investigators noted that further evaluation of the safety and clinical activity of this agent in both PD-L1-positive and PD-L1-negative metastatic triple-negative breast cancer is ongoing.

In closing, Dr. Telli related that 'proof-of-concept' has been demonstrated and that immunotherapy approaches may prove to be relevant for women with triple-negative and BRCA1/2 mutation-posi- tive breast cancer. Additional studies of both pembrolizumab and MPDL3280A in advanced triplenegative breast cancer are planned for 2015, and Dr. Telli highlighted the urgent need for clinical trials of immune therapies in this setting. As with platinum, biomarkers of response will be vital to best select patients for this type of treatment approach.

\section{References}

1. Von Minckwitz G, Schneeweiss A, Loibl S, et al. Neoadjuvant carboplatin in patients with triple-negative and HER2-positive early breast cance (GeparSixto; GBG 66): a randomized phase 2 trial. Lancet Oncol 2014; 15:747-756.

2. Sikov WM, Berry DA, Perou CM, et al. Impact of the addition of carboplatin and/or bevacizumab to neoadjuvant once-per-week paclitaxel followed by dose-dense doxorubicin and cyclophosphamide on pathology complete response rates in stage II to III triple-negative breast cancer: CALGB 40603 (Aliance). J Clin Oncol 2015;33:13-21.

3. Von Minckwitz G, Hahnen E, Fasching PA, et al. Pathological complete response rates after carboplatin-containing neoadjuvant chemotherapy in patients with germline BRCA mutation and triple-negative breast cancer: results from GeparSixto [abstract]. J Clin Oncol 2014;32(Suppl):Abstract 1005.

4. Telli ML, Jensen KC, Kurian AW, et al. PrECOG 0105: final efficacy results from a phase II study of gemcitabine and carboplatin plus iniparib as neoadjuvant therapy for triple-negative and BRCA1/2 mutation-associated breast cancer [abstract]. J Clin Oncol 2013;31(Suppl):Abstract 1003.

5. Tutt A, Ellis P, Kilburn L, et al. The TNT trial: a randomized phase III trial of carboplatin compared with docetaxel for patients with metastatic or recurrent locally advanced triple negative of BRCA1/2 breast cancer (CRUK/07/012) [abstract]. Presented at the 2014 San Antonio Breast Cancer Symposium; December 9-13, 2014; San Antonio, Texas. Abstract S3-01.

6. Nanda R, Chow LQ, Dees EC, et al. A phase Ib study of pembrolizumb (MK-3475) in patients with advanced triple-negative breast cancer [abstract]. Presented at the 2014 San Antonio Breast Cancer Symposium; December 9-13, 2014; San Antonio, Texas. Abstract S1-09.

7. Emens LA, Braiteh FS, Cassier P, et al. Inhibition of PD-L1 by MPDL $3280 A$ leads to clinical activity in patients with metastatic triple-negative breast cancer [abstract]. Presented at the 2014 San Antonio Breast Cancer Symposium; December 9-13, 2014; San Antonio, Texas. Abstract PD1-6. 